\title{
事业单位数字化档案管理现状及对策探讨
}

\author{
孟菳 \\ 洮南市劳动保障事务所
}

DOI:10.32629/ej.v2i6.270

\begin{abstract}
[摘 要] 对于事业单位来讲,档案管理工作是其日常管理工作的重要组成部分,档案作为事业单位日常工作中不断积累的相关资料,其对于 单位未来发展产生较大的影响, 如果我们的档案管理工作质量偏低, 那么档案本身的价值就无法有效发挥,且档案管理效率会大大下降, 这对 于事业单位的整体发展是非常不利的。事业单位档案具有数量多类型复杂的特征, 尤其是在当前社会发展速度较快的情况下, 我们每天的工 作中都会产生各种不同类型的档案资料,这些档案资料不仅数量多而且涉及范围广,所以事业单位的档案管理工作必须进行现代化建设,数 字化档案管理工作正是在这一形势下被提出的,数字化档案管理工作有效利用了计算机技术以及数字化技术, 应用专业档案管理程序对大量 档案资料进行科学管理,这是满足当前事业单位档案管理需求的主要发展方向,本文将针对事业单位数字化档案管理工作现状以及对策进行 分析。
\end{abstract}

[关键词] 事业单位; 数字化档案管理; 现状及对策

由于事业单位自身档案内容数量较多种类繁杂, 因此传统档案管理 形式难以满足当前档案管理需求, 我们必须针对档案管理工作进行现代 化建设, 在这一建设目标的指引下, 数字化档案管理工作的理念被确立, 在深入应用计算机数字化技术的情况下以计算机及相关档案管理系统 来替代人工操作, 满足档案管理工作效率以及质量上的要求, 从当前事 业单位档案管理工作的现状上来看, 我们还存在较多的不足, 这些问题 在传统档案管理向数字化档案管理工作迈进的过程中是无可避免的, 但 是我们应该针对当前档案管理工作现状来进行充分分析, 提出一系列解 决问题的对策, 这样我们才能有效保障事业单位档案管理工作稳定向前 发展。

\section{1 事业单位数字化档案管理现状}

1.1专业人才缺失

当前多数事业单位的数字化档案管理工作都会存在工作人员专业 素质相对较低的问题, 由于在相当长一段时间内, 事业单位的档案管理 工作都沿用传统档案管理模式, 在工作中依靠人力来完成档案的分类、 保存、调取以及使用工作, 因此多数档案管理工作人员对于数字化档案 管理是比较陌生的, 从管理理念上来讲, 传统档案管理模式在操作上和 理解难度上都比较低, 工作开展比较简单, 但是在现代化档案管理工作 中, 数字化档案管理工作不仅在工作模式上较以往有较大差异, 同时在 管理理念以及质量控制等方面也有很大的不同, 传统档案管理工作人员 多依靠自身经验来完成相应工作, 但是在数字化档案管理过程中我们必 须掌握相关数字化技术, 了解档案管理系统的操作, 这些都需要档案管 理工作人员去慢慢熟悉, 在数字化档案管理工作中绝大多数内容对于传

首先, 工作作风与资源型城市转型发展速度、质量息息相关。城市发 展中, 需保障各项措施落实到实处, 并从小事做起。所以, 资源型城市转型 发展中, 需做好顶层设计时, 并落实到位。其次, 建立完善考核、问责长效 机制。一是围绕资源型城市转型发展, 营造良好工作氛围, 提高工作效率与 工作质量。二是始终以人民利益为根本, 实事求是落实相关政策。三是增 强服务意识与服务水平, 坚决反对形式主义等不良行为, 真实为民众服务。

\section{4 结语}

总之, 资源型城市经济发展主要以资源消耗发展经济, 受国内经济增 速放缓、宏观经济政策等影响, 资源型城市转型发展尤为重要。所以, 资源 型城市转型发展期间, 需重视优化产业结构, 产业转型发展等措施的落实,
统档案管理人员来讲都是陌生的, 这也就导致很多事业单位档案部门在 数字化档案管理工作开展过程中整体效果不佳, 虽然一些单位积极引入 相关专业人才, 但是技术下行开展较慢很难在短时间内向全体员工进行 大范围的辐射, 因此专业人才缺失是当前事业单位数字化档案管理工作 现状之一, 也是主要问题 ${ }^{[1]}$ 。

\section{2 数字化技术应用存在问题}

对于数字化档案管理工作来讲, 数字化技术的应用自然是其中的重 点部分, 但是在实际进行相关工作的过程中, 我们发现, 多数事业单位在 数字化技术应用过程中仍存在较大问题, 首先是数字化技术应用不完全 的问题, 由于很多事业单传统档案资料内容数量庞大, 所以档案数字化 工作的推进非常缓慢, 很多原有的传统档案资料还来不及转换为数字化 档案, 在日常工作开展过程中只能保障当下发生的各种档案工作以数字 化形式进行处理。其次, 很多单位在档案管理工作中数字化技术应用比 较落后, 没有根据当前最新档案管理理念来建立相应的数字化档案管理 体系, 导致了相关工作开展过程中技术与实际需求不匹配, 我们在档案 管理工作中应用数字化技术的初衰就是提升档案管理工作的效率以及 质量, 但是这样的问题却严重影响了事业单位档案管理工作的开展, 与 数字化档案管理工作的优势发生了冲突, 最终导致工作效率以及工作质 量不理想 ${ }^{[2]}$ 。

1. 3缺乏科学的工作目标

档案管理工作如果没有科学明确的工作目标那么其管理质量一定 是比较低的, 在数字化档案管理工作中由于对新的工作模式还不熟悉所 以在具体的工作制度上对具体的工作目标制定是比较模糊的, 尚且没有对

推动资源型城市发展。

[参考文献]

[1]石绍云.资源型城市实现可持续发展所面临的问题与对策——以 山东省东营市为例[J].环渤海经济瞭望,2019(08):59-60.

[2]宁芳.辽宁省资源枯竭型城市转型尝试——以阜新经济转型初期 为例 [J].兰台世界,2019(08):4-5.

[3]谢士强,曹红辉.新时期资源型城市高质量转型发展之路——以河 北省武安市为例[J].中国西部,2019(04):25-35.

[4]找寻城市发展新资源北京大学相关研究报告显示,我国近一半资 源型城市面临严重转型问题[J].广西节能,2017(02):22-23. 
工作目标进行具体量化, 这样的情况下很多事业单位的档案管理工作就 失去了有效的管理体系支撑, 由于缺乏科学的工作目标因此很多工作人 员在工作过程中容易出现工作标准掌握不清晰的问题, 这影响了事业单 位档案管理工作的整体质量, 同时由于缺乏科学的工作目标, 很多工作 人员在相关技术应用以及工作评价上都出现了一系列问题, 由于工作目 标没有具体的量化指标所以在技术应用过程中很难做到科学应用, 而且 在实际工作过程中当前工作方式是否能够达到工作实际需求也不明确, 这种情况下我们的档案管理工作发展陷入了两难的境地。对于档案管理 工作来讲, 如果缺乏科学的工作目标, 那么其工作的安全性也得不到保 障, 存在资料外泄等一系列问题, 这对事业单位档案管理工作来讲是非 常危险的。

\section{2 事业单位数字化档案管理对策研究}

2.1积极引入专业人才并提升原有档案管理人员专业素质

由于数字化档案管理工作与传统档案管理工作模式发生了较大变 化, 因此我们必须有效提升工作人员的整体专业素质, 在达成这一目标 的过程中我们不仅要积极引入专业人才来加强数字化档案管理工作的 建设工作, 同时也要针对原有档案管理人员进行积极培训, 从整体上提 升档案管理工作人员的专业能力。在数字化档案管理工作建设过程中, 我们在人才引入过程中要重点针对专业对口人才来进行聘用, 当前我国 众多高校已经开设了档案管理相关专业, 这些专业人才对于档案数字化 管理有更加专业的技术掌握, 同时在工作过程中也能够更加准确的对数 字化档案管理工作进行设计执行。同时我们应该加强档案管理人员的专 业培训工作, 根据自身工作内容以及工作需求来进行相对应的专业培 训。在培训过程中我们可以依托自身专业人才来进行培训, 如果体系内 工作人员不能满足培训要求也可以通过聘请专业讲师以及其它档案管 理人员来满足相应的培训需求 ${ }^{[3]}$ 。

2.2 科学应用数字化技术

数字化档案管理工作在实际执行过程中仍然面临着技术应用不合 理的情况, 这一情况反映在具体工作内容上可以表现为档案管理效率不 高, 整体管理质量较低以及档案价值发挥不彻底等, 我们在现代化事业 单位档案管理工作中不仅应该重视数字化档案技术的引入工作, 更应该 了解数字化档案管理技术充分应用技术优势来实现提升整体工作质量 的目标。首先在档案录入、档案存储、档案调取操作中, 我们应该根据 单位自身工作需求以及档案类型对操作界面可设置内容进行妥善的设
计, 使之更加符合我们日常工作需求, 同时在处理各种档案问题的过程 中我们还应该重视档案管理操作的安全性, 事业单位档案在依托数字化 技术进行管理操作的过程中必然要使用到相应的网络体系, 档案资料在 网络体系中进行传递的过程中就必然面临着安全风险, 我们应该根据档 案级别来对档案管理权限进行严格的限制, 并且在防火墙设置上提升安 全等级, 保障整体档案资料的安全性。最后, 在数字化档案管理工作中, 我们应该重视档案价值的提取工作, 明确档案管理工作绝不是单纯保管 档案这么简单这一道理, 在档案管理过程中利用大数据计算以及云储存 等技术来实现档案价值的最大化。

2.3制定科学的工作目标

我们在进行数字化档案管理工作的过程中, 工作目标相较于以往自然 应该有所改变, 至少在管理效率以及管理效果上都应该有明显的提升, 因 此为了有增强档案管理工作的整体质量, 我们必须制定符合当前实际情况 的工作目标, 保障档案管理工作的规范性, 同时在工作过程中应该根据工 作目标来进行相应的管理工作。在工作目标的制定上, 我们首先要满足多 种文档格式的存储调取工作, 这也是数字化档案管理工作相较于传统模式 的最主要区别, 应该满足一份档案资料中可容纳多种不同资料格式的要求, 这对于数字化档案管理工作来讲是最基本的。同时在管理效率上应该所有 保障, 确立档案调取使用的具体流程以及整体耗时, 在工作过程中应该严 格按照相应标准来完成档案管理任务 ${ }^{[4]}$

\section{3 结束语}

事业单位的档案管理工作对于其长期稳定发展具有重要意义, 档案资 料是历史的见证, 是工作过往的具体体现, 我们想要在未来获得更好的发 展空间那么就一定要不断提升档案管理工作质量。数字化档案管理作为现 代档案管理工作的核心其相较于传统档案管理模式自然不必多说, 我们应 该充分利用现代技术, 有效实现事业单位档案管理工作的高质高效运行。

\section{[参考文献]}

[1]余晓芬.事业单位数字化档案管理现状及对策探讨 [J].科技 风,2019,(26):213.

[2]余雯婕.新形势下事业单位数字化档案管理策略[J].决策与信息(中 旬刊),2016,(12):211.

[3]张晓斌.机关事业单位数字化档案管理现状与优化对策[J].办公室 业务,2016,(6):132.

[4]徐明芳.事业单位数字化档案管理现状与优化对策[J].卷宗,2016,(7):99. 